\title{
Backstepping Control for a Tandem Rotor UAV Robot with Two 2-DOF Tiltable Coaxial Rotors
}

\author{
Xiongshi $\mathrm{Xu}{ }^{1}$, Keigo Watanabe ${ }^{2}$, Isaku Nagai ${ }^{3}$ \\ 1, 2,3 Graduate School of Natural Science and Technology, Okayama University, Okayama, Japan \\ ${ }^{2}$ BAICIRS, Beijing Institute of Technology (BIT), Beijing, China \\ Email: ${ }^{1}$ pyaj2yf4@s.okayama-u.ac.jp, ${ }^{2,3}$ \{watanabe, in\}@ sys.okayama-u.ac.jp
}

\begin{abstract}
The study of a fully actuated multi-rotor UAV robot is very important in the field of infrastructure inspection, because it needs a dexterous motion, such as a hovering in a special fixed attitude etc. This paper presents a backstepping control method for a simplified fully actuated model of a tandem rotor UAV robot with two 2 -DOF tiltable coaxial rotors. An MIMO vectorial backstepping approach is adopted here, because the input distribution matrix is a square and nonsingular matrix. The two-stage control method based on the Lyapunov second method is presented to stabilize the position and attitude of the whole system. The static control allocation problem is also solved by using a Moore-Penrose pseudoinverse. Finally, two simulations are demonstrated to verify the performance of the proposed control method, where one is a stabilizing problem in which all the desired position and attitude are to be constant, whereas the other is a trajectory tracking problem in which the desired positions are time-varying while the desired attitudes are to be constant.
\end{abstract}

Keywords-tiltable coaxial rotor, UAV, control allocation, backstepping control

\section{INTRODUCTION}

Nowadays, multi-rotor unmanned aerial vehicle (UAV) robots are going to be applied for human transportation [1], delivery of goods [2], [3], search and rescue application [4], [5], infrastructure inspection [6], [7], precision agriculture [8], [9], photogrammetry and mapping [10], [11], [12], etc. However, all the rotors of conventional multi-rotor type UAV robots are fixed to the fuselage in a single plane [13], which constrains the thrust generated by the propellers and its translational and rotational movements are coupled and cannot be controlled independently. In addition, it is difficult for conventional multi-rotor type UAV robots to maintain a stable flight in the proximity inspections of bridges and buildings.

Motivated by the above requirements and to try to control the translational and rotational movements of the aerial robot independently, a fully actuated, or redundant actuated multirotor UAV robot concept [14], [15], has been already carried out to decouple the position and attitude control of the robots by introducing a tiltable rotor mechanism [16], [17], or fixed tilt rotor mechanism [18], [19], [20].

However, in most cases, the number of motors including the tilt actuators is generally over 6 . For example, Allenspach et al. [21] introduced a 1-DOF tilt mechanism for each coaxial rotor in a hexarotor equipped 18 motors in total, where they derived a 6-DOF optimal controller with an actuator allocation approach to implement the task prioritization. Segui-Gasco et al. [22] proposed to use a 2DOF tilt mechanism for each rotor in a quadrotor, in which 12 motors were mounted. The control system they designed was based on the classical control theory and pseudoinverse control allocation. As a result, most of control systems in multi-rotor UAV robots with tilt mechanisms become a socalled redundant actuated system, so that there have some redundant DOFs in kinematics to a generalized force for a given 6-DOF space motion. Solving the rotation speed and tilt angle of each tilt rotor simultaneously and uniquely is generally impossible for such a redundant actuated system.

Therefore, $\mathrm{Xu}$ et al. [23] already proposed a tandem rotor UAV robot in which a tilt rotor mechanism with 2-DOF tilt angles per rotor [24], [25] was arranged in front of and behind the fuselage, where a coaxial rotor mechanism [26], [27], [28] was used so as to cancel the anti-torque of the rotor. Hence, the system model of the UAV robot can be simplified. The proposed tandem rotor UAV robot was examined to fly in the air, or to travel on the ground and wall, aiming to provide a stable flight for infrastructure inspection or for other applications that need high stability.

In this study, an MIMO vectorial backstepping control method is applied for designing the control inputs for the three forces and three torques in 6-DOF motions, because the input distribution matrix is a square and nonsingular matrix that can be invertible for such a generalized vector for thrust forces and torques. Some simulation results of the proposed UAV robot are given to demonstrate the decoupling control of the position and attitude and the tracking performance of arbitrary position and attitude.

In what follows, Section II introduces the dynamical models of the proposed UAV robot, together with giving a solution to a static control allocation problem by using a Moore-Penrose pseudo-inverse technique. Section III presents the proposed backstepping control method for the UAV robot. Some simulation results are given to show the tracking ability of decoupling the position and attitude of the UAV robot in Section IV. Finally, conclusions are drawn in Section V. 


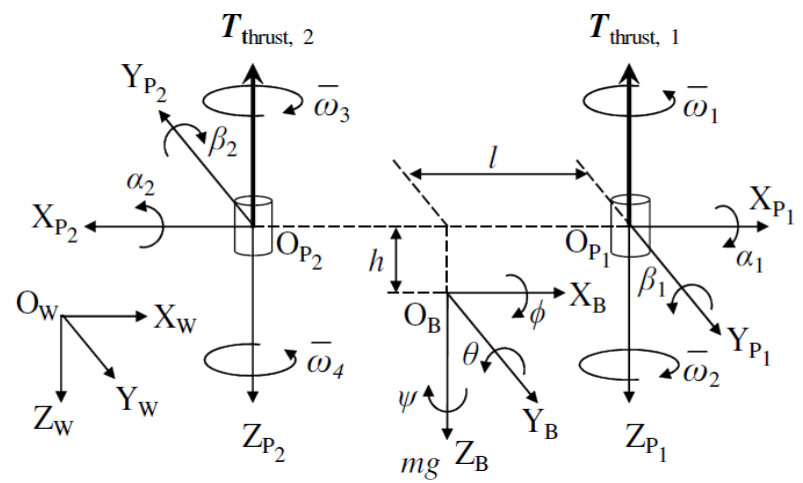

Fig. 1. Coordinate systems related to a tandem rotor UAV robot

\section{DYNAMICAL MODELS}

Several coordinate systems (or frames) related to the tandem rotor UAV robot with two 2-DOF tiltable coaxial rotors are shown in Fig. 1. A 3D model of the proposed prototype UAV robot is also shown in Fig. 2.

The world frame $\mathcal{F}_{\mathrm{w}}$ is defined with the origin $\mathrm{O}_{\mathrm{w}}$ following the right-hand coordinate system with the axes $\left\{X_{w}, Y_{w}, Z_{w}\right\}$. The body frame $\mathcal{F}_{B}$ is defined with the center $\mathrm{O}_{\mathrm{B}}$ fixed to its center of mass with the axes $\left\{X_{B}, Y_{B}, Z_{B}\right\}$. The first tiltable coaxial rotor coordinate frame $\mathcal{F}_{\mathrm{P}_{1}}$ is defined with origin $\mathrm{O}_{\mathrm{P}_{1}}$ with the axes $\left\{\mathrm{X}_{\mathrm{P}_{1}}, \mathrm{Y}_{\mathrm{P}_{1}}, \mathrm{Z}_{\mathrm{P}_{1}}\right\}$, while the second tiltable coaxial rotor coordinate frame $\mathcal{F}_{\mathrm{P}_{2}}$ is defined with origin $\mathrm{O}_{\mathrm{P}_{2}}$ with the axes $\left\{\mathrm{X}_{\mathrm{P}_{2}}, \mathrm{Y}_{\mathrm{P}_{2}}, \mathrm{Z}_{\mathrm{P}_{2}}\right\}$.

For the convenience, the coordinate frame of the $i$-th $(i=1,2)$ tiltable coaxial rotor is unified to $\mathcal{F}_{\mathrm{P}_{i}}$. The rotation angles around the $\mathrm{X}_{\mathrm{B}^{-}}, \mathrm{Y}_{\mathrm{B}^{-}}$and $\mathrm{Z}_{\mathrm{B}}$-axis in $\mathcal{F}_{\mathrm{B}}$ are defined as $(\phi, \theta, \psi)$, while the tilt angles around the $\mathrm{Y}_{\mathrm{P}_{i}}$ - and $\mathrm{X}_{\mathrm{P}_{i}}$-axis in $\mathcal{F}_{\mathrm{P}_{i}}$ are defined as $\left(\beta_{i}, \alpha_{i}\right)$. The range of the tilt angle $\alpha_{i}$ is set to $\left[-\frac{\pi}{2}, \frac{\pi}{2}\right]$, and the range of the tilt angle $\beta_{i}$ is set to $[-\pi, \pi]$. The rotation matrix ${ }^{w} \boldsymbol{R}_{B}=\boldsymbol{R}_{Z}(\psi) \boldsymbol{R}_{Y}(\theta) \boldsymbol{R}_{X}(\phi)$ represents the rotation from $\mathcal{F}_{B}$ to $\mathcal{F}_{\mathrm{W}}$, in which $\boldsymbol{R}_{Z}, \boldsymbol{R}_{Y}$ and $\boldsymbol{R}_{X}$ are the rotations around the $\mathrm{Z}_{\mathrm{B}}-, \mathrm{Y}_{\mathrm{B}}-$ and $\mathrm{X}_{\mathrm{B}}$-axis, respectively. Also, ${ }^{B} \boldsymbol{R}_{P_{i}}=\boldsymbol{R}_{Z}((i-1) \pi) \boldsymbol{R}_{Y}\left(\beta_{i}\right) \boldsymbol{R}_{X}\left(\alpha_{i}\right)$ represents the rotation matrix from $\mathcal{F}_{\mathrm{P}_{i}}$ to $\mathcal{F}_{\mathrm{B}}$, in which $\boldsymbol{R}_{Z}$, $\boldsymbol{R}_{Y}$ and $\boldsymbol{R}_{X}$ are the rotations around the $\mathrm{Z}_{\mathrm{P}_{i}}{ }^{-}, \mathrm{Y}_{\mathrm{P}_{i}}{ }^{-}$and $\mathrm{X}_{\mathrm{P}_{i}}{ }^{-}$ axis, respectively. The length $l$ is the distance between the origin of each coaxial rotor $\mathrm{O}_{\mathrm{P}_{\mathrm{i}}}$ and the axis $\mathrm{Z}_{\mathrm{B}}$ of the UAV robot, while the height $h$ is the distance between the origin of $\mathrm{O}_{\mathrm{P}_{\mathrm{i}}}$ and $\mathrm{O}_{\mathrm{B}}$ in $\mathrm{Z}_{\mathrm{B}}$ direction. The position vector $\boldsymbol{O}_{P_{i}}$ of the $i$ th tiltable coaxial rotor in $\mathcal{F}_{\mathrm{B}}$ can be defined as ${ }^{B} \boldsymbol{O}_{P_{i}}=$ $\boldsymbol{R}_{Z}((i-1) \pi)\left[\begin{array}{lll}l & 0 & h\end{array}\right]^{T}$. As shown in Fig. $1, \bar{\omega}_{1}, \bar{\omega}_{2}, \bar{\omega}_{3}$ and $\bar{\omega}_{4}$ denote the rotation speed of the brushless motors in each coaxial rotor, respectively. Hence, the anti-torque $\boldsymbol{\tau}_{\mathrm{drag}, i}$ of the $i$-th coaxial rotor can be given by

$$
\left\{\begin{array}{l}
\boldsymbol{\tau}_{\mathrm{drag}, 1}=\left[\begin{array}{lll}
0 & 0 & k_{m}\left(\bar{\omega}_{2}^{2}-\bar{\omega}_{1}^{2}\right)
\end{array}\right]^{T} \\
\boldsymbol{\tau}_{\mathrm{drag}, 2}=\left[\begin{array}{lll}
0 & 0 & k_{m}\left(\bar{\omega}_{3}^{2}-\bar{\omega}_{4}^{2}\right)
\end{array}\right]^{T}
\end{array}\right.
$$

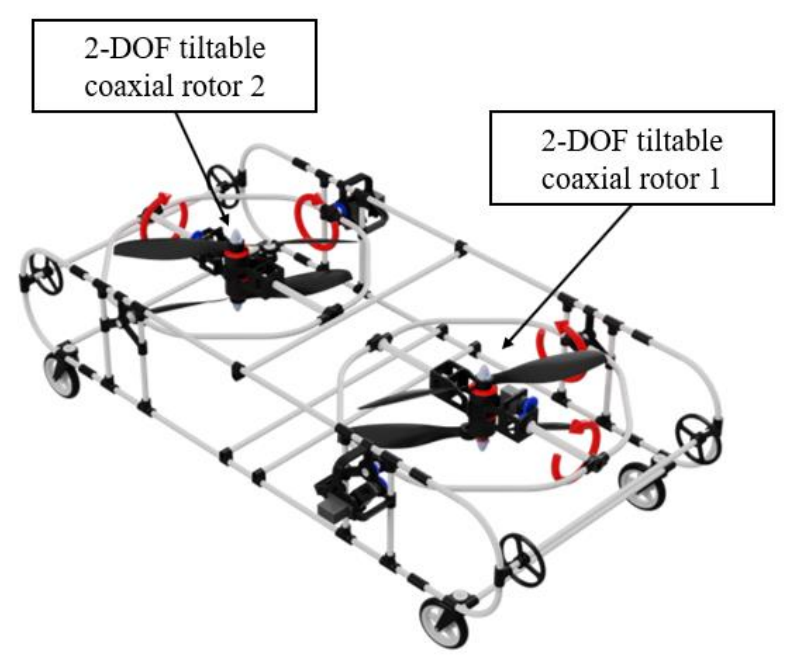

Fig. 2. 3D model of the proposed UAV robot

where $k_{m}$ is the propeller drag coefficient and $k_{m}>0$. Also, the total output thrust $\boldsymbol{T}_{\text {thrust }, i}$ of each coaxial rotor is given by

$$
\left\{\begin{array}{l}
\boldsymbol{T}_{\text {thrust, } 1}=\left[\begin{array}{lll}
0 & 0 & -k_{f}\left(\bar{\omega}_{2}^{2}+\bar{\omega}_{1}^{2}\right)
\end{array}\right]^{T} \\
\boldsymbol{T}_{\text {thrust, } 2}=\left[\begin{array}{lll}
0 & 0 & -k_{f}\left(\bar{\omega}_{3}^{2}+\bar{\omega}_{4}^{2}\right)
\end{array}\right]^{T}
\end{array}\right.
$$

in which $k_{f}$ is the propeller thrust coefficient and $k_{f}>0$.

For the simplify of the control model, the anti-torque $\boldsymbol{\tau}_{\text {drag }, i}$ of the $i$-th tiltable coaxial rotor can be eliminated by setting $\bar{\omega}_{1}=\bar{\omega}_{2}=\omega_{1}, \bar{\omega}_{3}=\bar{\omega}_{4}=\omega_{2}$, where $\omega_{1}$ and $\omega_{2}$ are the new rotation speeds for the brushless motors in each tiltable coaxial rotor. Hence, the anti-torque $\boldsymbol{\tau}_{\text {drag }}$ can be obtained as

$$
\boldsymbol{\tau}_{\text {drag }}=\boldsymbol{\tau}_{\text {drag, } 1}+\boldsymbol{\tau}_{\text {drag, } 2}=\mathbf{0}
$$

Also, the gyro moment effect of the tiltable coaxial rotors can be ignored by un-modeling the tilt angular velocities and accelerations of them.

\section{A. Dynamical equations in rotation}

From the previous study [29], $\boldsymbol{\omega}^{B} \triangleq\left[\begin{array}{lll}\dot{\phi} & \dot{\theta} & \dot{\psi}\end{array}\right]^{T}$ is the angular velocity of the UAV robot expressed in the body coordinate system. Under the Newton-Eulerian's law, $\boldsymbol{\omega}^{B}$ is subject to

$$
\boldsymbol{I}_{B} \dot{\boldsymbol{\omega}}^{B}+\boldsymbol{\omega}^{B} \times \boldsymbol{I}_{B} \boldsymbol{\omega}^{B}=\boldsymbol{\tau}^{B}+\boldsymbol{\tau}_{\mathrm{ext}}
$$

where $\boldsymbol{I}_{B}=\operatorname{diag}\left(I_{B_{x x}}, I_{B_{y y}}, I_{B_{z z}}\right)$ is the symmetric and positive definite inertia matrix of the body, $\boldsymbol{\tau}^{B}$ is defined as the input torque, and $\boldsymbol{\tau}_{\text {ext }}$ refers to the unmodeled disturbance. By ignoring the effect of unmodeled disturbance etc., $\boldsymbol{\tau}_{\text {ext }}=$ 0 , whereas the input torque $\boldsymbol{\tau}^{B}$ is defined as:

$$
\boldsymbol{\tau}^{B}=\boldsymbol{\tau}_{\text {thrust }}+\boldsymbol{\tau}_{\text {drag }}=\boldsymbol{\tau}_{\text {thrust }}
$$

in which the moment $\boldsymbol{\tau}_{\text {thrust }}$ due to the thrusts generated by the tiltable coaxial rotors is 


$$
\boldsymbol{\tau}_{\text {thrust }}=\sum_{i=1}^{2}\left({ }^{B} \boldsymbol{O}_{P_{i}} \times{ }^{B} \boldsymbol{R}_{P_{i}} \boldsymbol{T}_{\text {thrust }, i}\right)
$$

Therefore, denoting the resultant input torque as $\boldsymbol{\tau}^{B}=$ $\left[\begin{array}{ccc}\tau_{x}^{B} & \tau_{y}^{B} & \tau_{z}^{B}\end{array}\right]^{T}$, the dynamical model of the rotational motion of the UAV robot can be obtained as

$$
\left[\begin{array}{c}
\ddot{\phi} \\
\ddot{\theta} \\
\ddot{\psi}
\end{array}\right]=\left[\begin{array}{l}
\dot{\theta} \dot{\psi}\left(\frac{I_{B_{y y}}-I_{B_{z z}}}{I_{B_{x x}}}\right)+\frac{1}{I_{B_{x x}}} \tau_{x}^{B} \\
\dot{\phi} \dot{\psi}\left(\frac{I_{B_{z z}}-I_{B_{x x}}}{I_{B_{y y}}}\right)+\frac{1}{I_{B_{y y}}} \tau_{y}^{B} \\
\dot{\phi} \dot{\theta}\left(\frac{I_{B_{x x}}-I_{B_{y y}}}{I_{B_{z z}}}\right)+\frac{1}{I_{B_{z z}}} \tau_{z}^{B}
\end{array}\right]
$$

\section{B. Dynamical equations in translation}

According to the Newton equation of motion, the robot body position $\boldsymbol{P}=\left[\begin{array}{lll}x & y & z\end{array}\right]^{T}$ in the world coordinate system is subject to

$$
m \ddot{\boldsymbol{P}}=m\left[\begin{array}{l}
0 \\
0 \\
g
\end{array}\right]+{ }^{w} \boldsymbol{R}_{B} \sum_{i=1}^{2}{ }^{B} \boldsymbol{R}_{P_{i}} \boldsymbol{T}_{\text {thrust }, i}+\boldsymbol{f}_{\mathrm{ext}}
$$

where $\boldsymbol{f}_{\text {ext }}$ includes disturbances and unmodeled factors and $g$ is the gravity constant. By ignoring the influence of friction etc., $\boldsymbol{f}_{\text {ext }}$ can be set to 0 . Denoting the input thrust $\boldsymbol{f}^{B}$ in $\mathcal{F}_{\mathrm{B}}$ as

$$
\boldsymbol{f}^{B}=\sum_{i=0}^{2}{ }^{B} \boldsymbol{R}_{P_{i}} \boldsymbol{T}_{\text {thrust }, i}=\left[\begin{array}{lll}
F_{x}^{B} & F_{y}^{B} & F_{z}^{B}
\end{array}\right]^{T}
$$

the dynamical model of the translational motion of the UAV robot is expressed by

$$
\left[\begin{array}{c}
\ddot{x} \\
\ddot{y} \\
\ddot{z}
\end{array}\right]=\left[\begin{array}{l}
0 \\
0 \\
g
\end{array}\right]+\frac{1}{m}{ }^{w} \boldsymbol{R}_{B}\left[\begin{array}{l}
F_{x}^{B} \\
F_{y}^{B} \\
F_{z}^{B}
\end{array}\right]
$$

\section{Dynamical equations of the system}

Combining the equations (7) and (10), the dynamical model of the system in matrix form can be reduced to:

$$
\ddot{\boldsymbol{X}}=\boldsymbol{f}(\dot{\boldsymbol{X}})+\boldsymbol{g}(\boldsymbol{X}) \boldsymbol{U}
$$

where $\quad \boldsymbol{X}=\left[\begin{array}{llllll}x & y & z & \phi & \theta & \psi\end{array}\right]^{T}$ is a generalized coordinate vector related to the proposed UAV robot, $\boldsymbol{U}=$ $\left[\begin{array}{llllll}F_{x}^{B} & F_{y}^{B} & F_{z}^{B} & \tau_{x}^{B} & \tau_{y}^{B} & \tau_{z}^{B}\end{array}\right]^{T}=\left[\begin{array}{lll}\left(\boldsymbol{f}^{B}\right)^{T} & \left(\boldsymbol{\tau}^{B}\right)^{T}\end{array}\right]^{T} \quad$ is $\quad$ a vector of the thrust forces and torques of the coaxial rotors expressed in the body coordinate system on $\mathrm{X}_{\mathrm{B}^{-}}, \mathrm{Y}_{\mathrm{B}^{-}}$and $\mathrm{Z}_{\mathrm{B}^{-}}$ axis. Here, $\boldsymbol{f}(\dot{\boldsymbol{X}})$ and $\boldsymbol{g}(\boldsymbol{X})$ can be rewritten as

$$
\begin{aligned}
& \boldsymbol{f}(\dot{\boldsymbol{X}})=\left[\begin{array}{c}
0 \\
0 \\
g \\
\dot{\theta} \dot{\psi}\left(\frac{I_{B_{y y}}-I_{B_{z z}}}{I_{B_{x x}}}\right) \\
\dot{\phi} \dot{\psi}\left(\frac{I_{B_{z z}}-I_{B_{x x}}}{I_{B_{y y}}}\right) \\
\dot{\phi} \dot{\theta}\left(\frac{I_{B_{x x}}-I_{B_{y y}}}{I_{B_{z z}}}\right)
\end{array}\right] \\
& \boldsymbol{g}(\boldsymbol{X})=\left[\begin{array}{cc}
\frac{1}{m}{ }^{w} \boldsymbol{R}_{B} & \mathbf{0} \\
\mathbf{0} & \boldsymbol{M}_{\boldsymbol{I}_{B}}^{-1}
\end{array}\right]
\end{aligned}
$$

in which $\boldsymbol{g}(\boldsymbol{X}) \in \mathbb{R}^{6 \times 6}$ and $\boldsymbol{M}_{\boldsymbol{I}_{B}}=\operatorname{diag}\left(I_{B_{x x}}, I_{B_{y y}}, I_{B_{z z}}\right)$. It is found that $\boldsymbol{g}^{-1}(\boldsymbol{X})$ exists and it can be obtained as

$$
\boldsymbol{g}^{-1}(\boldsymbol{X})=\left[\begin{array}{cc}
m^{w} \boldsymbol{R}_{B}{ }^{T} & \mathbf{0} \\
\mathbf{0} & \boldsymbol{M}_{\boldsymbol{I}_{B}}
\end{array}\right]
$$

because the rotation matrix ${ }^{w} \boldsymbol{R}_{B}$ is orthogonal, i.e., ${ }^{w} \boldsymbol{R}_{B}{ }^{-1} \equiv{ }^{w} \boldsymbol{R}_{B}{ }^{T}, I_{B_{x x}} \neq 0, I_{B_{y y}} \neq 0$ and $I_{B_{z z}} \neq 0$. Hence, the whole system can be simplified to a fully actuated system which has six inputs and six outputs (i.e., generalized coordinate variables). The input thrust $\boldsymbol{f}^{B}$ and input torque $\boldsymbol{\tau}^{B}$ can be obtained as below:

$$
\left[\begin{array}{c}
\boldsymbol{f}^{B} \\
\boldsymbol{\tau}^{B}
\end{array}\right] \triangleq\left[\begin{array}{c}
-2 k_{f} a_{1}+2 k_{f} a_{2} \\
2 k_{f} a_{3}-2 k_{f} a_{4} \\
-2 k_{f} a_{5}-2 k_{f} a_{6} \\
-2 h k_{f} a_{3}+2 h k_{f} a_{4} \\
2 l k_{f} a_{5}-2 l k_{f} a_{6}-2 h k_{f} a_{1}+2 h k_{f} a_{2} \\
2 l k_{f} a_{3}-2 l k_{f} a_{4}
\end{array}\right]
$$

in which $a_{1}=\cos \alpha_{1} \sin \beta_{1} \omega_{1}^{2}, a_{2}=\cos \alpha_{2} \sin \beta_{2} \omega_{2}^{2}$, $a_{3}=\sin \alpha_{1} \omega_{1}^{2}, a_{4}=\sin \alpha_{2} \omega_{2}^{2}, a_{5}=\cos \alpha_{1} \cos \beta_{1} \omega_{1}^{2}$ and $a_{6}=\cos \alpha_{2} \cos \beta_{2} \omega_{2}^{2}$. By decomposing the angular velocity of the $i$-th coxial rotor into three contributions [30], [31], it can be written as $x_{i}^{a}=\cos \alpha_{i} \sin \beta_{i} \omega_{i}^{2}, x_{i}^{b}=\sin \alpha_{i} \omega_{i}^{2}$ and $x_{i}^{c}=\cos \alpha_{i} \cos \beta_{\mathrm{i}} \omega_{i}^{2}$, so that $\alpha_{i}=\sin ^{-1}\left(x_{i}^{b}, \omega_{i}^{2}\right), \beta_{i}=$ $\tan ^{-1}\left(x_{i}^{a}, x_{i}^{c}\right)$ and $\omega_{i}=\sqrt{\left(x_{i}^{a}\right)^{2}+\left(x_{i}^{b}\right)^{2}+\left(x_{i}^{c}\right)^{2}}$. Hence, the transformed input vector $\boldsymbol{x}$ is given by

$$
\boldsymbol{x}=\left[\begin{array}{llllll}
x_{1}^{a} & x_{1}^{b} & x_{1}^{c} & x_{2}^{a} & x_{2}^{b} & x_{2}^{c}
\end{array}\right]^{T}, \boldsymbol{x} \in \mathbb{R}^{6}
$$

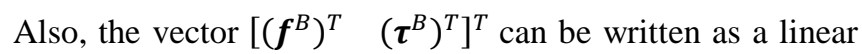

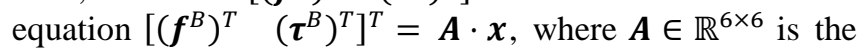
static control allocation matrix that maps the actuators to the given forces and torques, which can be reduced to

$$
\boldsymbol{A}=k_{f}\left[\begin{array}{cccccc}
-2 & 0 & 0 & 2 & 0 & 0 \\
0 & 2 & 0 & 0 & -2 & 0 \\
0 & 0 & -2 & 0 & 0 & -2 \\
0 & -2 h & 0 & 0 & 2 h & 0 \\
-2 h & 0 & 2 l & 2 h & 0 & -2 l \\
0 & 2 l & 0 & 0 & 2 l & 0
\end{array}\right]
$$

Note here that $\boldsymbol{A}$ is singular though it is a square matrix. Therefore, the force and torque can be resolved by using the Moore-Penrose pseudo-inverse of the static control allocation matrix $\boldsymbol{A}$, which can be expressed as below: 


$$
\boldsymbol{x}=\boldsymbol{A}^{\dagger}\left[\begin{array}{l}
\boldsymbol{f}^{B} \\
\boldsymbol{\tau}^{B}
\end{array}\right], \quad \boldsymbol{A}^{\dagger} \in \mathbb{R}^{6 \times 6}
$$

where it should be noted that $\boldsymbol{A}^{\dagger}$ is the minimum norm solution of $\boldsymbol{A}$. because $\boldsymbol{A}$ is singular though it is a square matrix. And the rank of $\boldsymbol{A}$ is 5 .

\section{BACKSTEPPING CONTROL METHOD}

The design of the backstepping control [32] is extended to a vector form in this paper. That is, a MIMO vectoral backstepping approach [33] is explained here. The detailed derivations are given step by step as follows:

1) Derivation about the first Lyapunov function:

When introducing a state variable representation,

$$
x_{1}=X, \quad x_{2}=\dot{X}
$$

the equation (11) can be written as

$$
\begin{aligned}
& \dot{x}_{1}=x_{2} \\
& \dot{x}_{2}=f\left(x_{2}\right)+g\left(x_{1}\right) U
\end{aligned}
$$

Introduce the position error as $\boldsymbol{e}_{1}=\boldsymbol{x}_{1 d}-\boldsymbol{x}_{1}$, where $\boldsymbol{x}_{1 d}$ is a desired reference trajectory. The first Lyapunov function is chosen as

$$
\boldsymbol{V}_{1}=\frac{1}{2} \boldsymbol{e}_{1}^{T} \boldsymbol{e}_{1}
$$

then the derivative of $\boldsymbol{V}_{1}$ with respect to time is

$$
\dot{\boldsymbol{V}}_{1}=\boldsymbol{e}_{1}^{T} \dot{\boldsymbol{e}}_{1}=\boldsymbol{e}_{1}^{T}\left(\dot{\boldsymbol{x}}_{1 d}-\dot{\boldsymbol{x}}_{1}\right)
$$

For stabilizing $\boldsymbol{e}_{1}$, a stabilizing function is designed as

$$
\boldsymbol{\alpha}_{1}=\dot{\boldsymbol{x}}_{1 d}+\boldsymbol{K}_{1} \boldsymbol{e}_{1}
$$

where $\boldsymbol{K}_{1}>0$. Substituting $\dot{\boldsymbol{x}}_{1 d}$ in $\dot{\boldsymbol{V}}_{1}$ by (23), $\dot{\boldsymbol{V}}_{1}$ can be obtained as

$$
\begin{aligned}
\dot{\boldsymbol{V}}_{1} & =\boldsymbol{e}_{1}^{T}\left(-\boldsymbol{K}_{1} \boldsymbol{e}_{1}+\boldsymbol{\alpha}_{1}-\dot{\boldsymbol{x}}_{1}\right) \\
& =\boldsymbol{e}_{1}^{T}\left(-\boldsymbol{K}_{1} \boldsymbol{e}_{1}+\boldsymbol{\alpha}_{1}-\boldsymbol{x}_{2}\right) \\
& =-\boldsymbol{e}_{1}^{T} \boldsymbol{K}_{1} \boldsymbol{e}_{1}+\boldsymbol{e}_{1}^{T} \boldsymbol{e}_{2}
\end{aligned}
$$

where $\boldsymbol{e}_{2}=\boldsymbol{\alpha}_{1}-\boldsymbol{x}_{2}$ denotes an extended velocity tracking error. When $\boldsymbol{e}_{2} \equiv \mathbf{0}, \dot{\boldsymbol{V}}_{1}=-\boldsymbol{e}_{1}^{T} \boldsymbol{K}_{1} \boldsymbol{e}_{1} \leq \mathbf{0}$ can be obtained.

2) Derivation about the second Lyapunov function: Introduce the velocity error again as

$$
\boldsymbol{e}_{2}=\boldsymbol{\alpha}_{1}-\boldsymbol{x}_{2}=\dot{x}_{1 d}-\dot{x}_{1}+\boldsymbol{K}_{1} \boldsymbol{e}_{1}
$$

then the derivative of $\boldsymbol{e}_{2}$ can be represented as

$$
\begin{aligned}
\dot{e}_{2} & =\dot{\alpha}_{1}-\dot{x}_{2} \\
& =\ddot{x}_{1 d}+K_{1} \dot{e}_{1}-\boldsymbol{f}\left(\boldsymbol{x}_{2}\right)-\boldsymbol{g}\left(\boldsymbol{x}_{1}\right) \boldsymbol{U}
\end{aligned}
$$

The second Lyapunov function is introduced as

$$
\boldsymbol{V}_{2}=\boldsymbol{V}_{1}+\frac{1}{2} \boldsymbol{e}_{2}^{T} \boldsymbol{e}_{2} \equiv \frac{1}{2} \boldsymbol{e}_{1}^{T} \boldsymbol{e}_{1}+\frac{1}{2} \boldsymbol{e}_{2}^{T} \boldsymbol{e}_{2}
$$

then the derivative of $\boldsymbol{V}_{2}$ with respect to time is

$$
\dot{\boldsymbol{V}}_{2}=\dot{\boldsymbol{V}}_{1}+\boldsymbol{e}_{2}^{T} \dot{\boldsymbol{e}}_{2}
$$

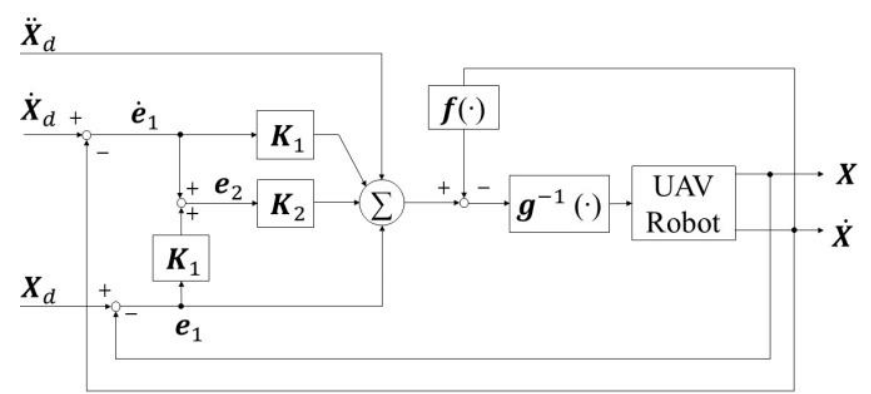

Fig. 3. Block diagram of the proposed backstepping control method

Combining the equations (21) and (23) mentioned above, $\dot{\boldsymbol{V}}_{2}$ can be obtained as

$$
\begin{aligned}
\dot{\boldsymbol{V}}_{2}= & -\boldsymbol{e}_{1}^{T} \boldsymbol{K}_{1} \boldsymbol{e}_{1}+\boldsymbol{e}_{1}^{T} \boldsymbol{e}_{2} \\
& +\boldsymbol{e}_{2}^{T}\left[\ddot{\boldsymbol{x}}_{1 d}+\boldsymbol{K}_{1} \dot{\boldsymbol{e}}_{1}-\boldsymbol{f}\left(\boldsymbol{x}_{2}\right)-\boldsymbol{g}\left(\boldsymbol{x}_{1}\right) \boldsymbol{U}\right]
\end{aligned}
$$

For stabilizing the $\boldsymbol{e}_{2}$, the control input $\boldsymbol{U}$ is given as

$$
\boldsymbol{U}=g^{-1}\left(x_{1}\right)\left[K_{2} e_{2}+\ddot{x}_{1 d}+K_{1} \dot{e}_{1}-f\left(x_{2}\right)+e_{1}\right]
$$

where $\boldsymbol{K}_{2}>0, \boldsymbol{g}^{-\mathbf{1}}\left(\boldsymbol{x}_{1}\right)$ is set to be known. Substituting (30) to (29), it follows that $\dot{\boldsymbol{V}}_{2}=-\boldsymbol{e}_{1}^{T} \boldsymbol{K}_{1} \boldsymbol{e}_{1}-\boldsymbol{e}_{2}^{T} \boldsymbol{K}_{2} \boldsymbol{e}_{2} \leq \mathbf{0}$. Thus, it is found that $\boldsymbol{e}_{1}$ and $\boldsymbol{e}_{2}$ converge to zero when $t \rightarrow \infty$, so that this controlled system assures the asymptotic stability. Fig. 3 shows the block diagram of the proposed backstepping control method.

\section{SimUlation RESUlTS}

In order to verify the performance of the designed backstepping control strategy, two cases (stability and trajectory tracking) are simulated on MATLAB. The physical parameters of the UAV robot used in the simulation are $m=1.2 \mathrm{~kg}, g=9.81 \mathrm{~m} / \mathrm{s}^{2}, l=0.2 \mathrm{~m}, h=0.05 \mathrm{~m}$ and $k_{f}=1.984$ $\times 10^{-7} \mathrm{~N} /(\mathrm{rpm})^{2}$. The control gains are chosen as $K_{11}=K_{12}$ $=K_{13}=1, K_{14}=K_{15}=K_{16}=2$, and $K_{21}=K_{22}=K_{23}=K_{24}=$ $K_{25}=K_{26}=1$. The sampling width of the simulations is set to $0.01 \mathrm{~s}$.

\section{A. Simulation 1: Stabilizing Problem}

In the simulation, the desired values of the position and attitude are set to $\boldsymbol{X}_{d}=\left[\begin{array}{llllll}0.5 & 1.0 & -1.5 & \frac{\pi}{6} & \frac{\pi}{6} & \frac{\pi}{6}\end{array}\right]^{T}$, the system is started at an initial state which is set to $\mathrm{X}_{0}=$ $\left[\begin{array}{llllll}0 & 0 & 0 & -\frac{\pi}{12} & -\frac{\pi}{12} & -\frac{\pi}{12}\end{array}\right]^{T}$.

As shown in Fig. 4, the positions of the UAV robot are converged to the desired values, where the rise time of the position responses in the $\mathrm{X}_{\mathrm{w}}{ }^{-}, \mathrm{Y}_{\mathrm{w}}{ }^{-}$and $\mathrm{Z}_{\mathrm{w}}$-axis are all about $1.52 \mathrm{~s}$. Their overshoots in the $\mathrm{X}_{\mathrm{w}}-, \mathrm{Y}_{\mathrm{w}}-$ and $\mathrm{Z}_{\mathrm{w}}$-axis are $0.0216 \mathrm{~m}, 0.0432 \mathrm{~m}$ and $-0.0648 \mathrm{~m}$, respectively. The settling time of the position responses in the $\mathrm{X}_{\mathrm{w}}^{-}, \mathrm{Y}_{\mathrm{w}^{-}}$and $\mathrm{Z}_{\mathrm{w}}$-axis are all about $3.85 \mathrm{~s}$. The Fig. 5 shows the attitude responses of the UAV robot are converged to the desired values, where the rise time of the attitude responses around the $\mathrm{X}_{\mathrm{w}}-, \mathrm{Y}_{\mathrm{w}}-$ and $\mathrm{Z}_{\mathrm{w}}-$ axis are all about $1.58 \mathrm{~s}$. Their overshoots around the $\mathrm{X}_{\mathrm{w}}{ }^{-}, \mathrm{Y}_{\mathrm{w}}-$ and $\mathrm{Z}_{\mathrm{w}}$-axis are all about 
$0.0036 \mathrm{rad}$. The settling time of the attitude responses in the $\mathrm{X}_{\mathrm{w}^{-}}, \mathrm{Y}_{\mathrm{w}^{-}}$and $\mathrm{Z}_{\mathrm{w}^{-}}$axis are all about $2.49 \mathrm{~s}$.

The time histories of the tilt angles during the simulation are shown in Fig. 6, where the changes between the steadystates and the current tilt angles $\alpha_{1}, \alpha_{2}, \beta_{1}$ and $\beta_{2}$ are reached to the maximum values at the starting $0.05 \mathrm{~s}$. It is also found, from this figure, that the rotational direction is different each other. Fig. 7 shows the rotational speed responses of the tiltable coaxial rotors, where the changes between the steadystates and the current values are reached to the maximum values at the beginning $0.05 \mathrm{~s}$. The stable rotation speeds of the motor in each coaxial rotor are also shown to be different.

From the simulation results, it can be seen that the proposed backstepping control method is useful for the decoupling the position and attitude control of the UAV robot. It should be noted that the actuators used in the tilt mechanisms and the coaxial rotors may not respond to such speeds in reality, because the dynamics of them are ignored in this simulation.
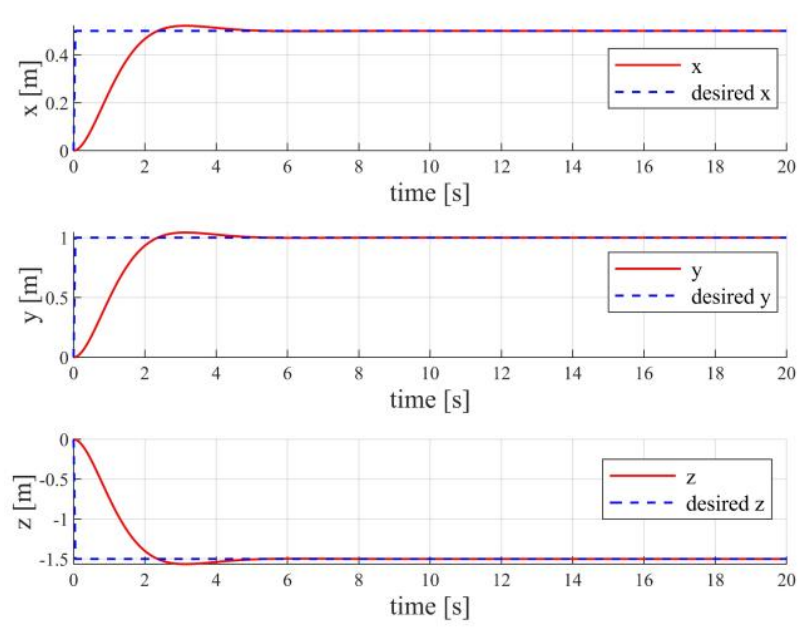

Fig. 4. Position control responses
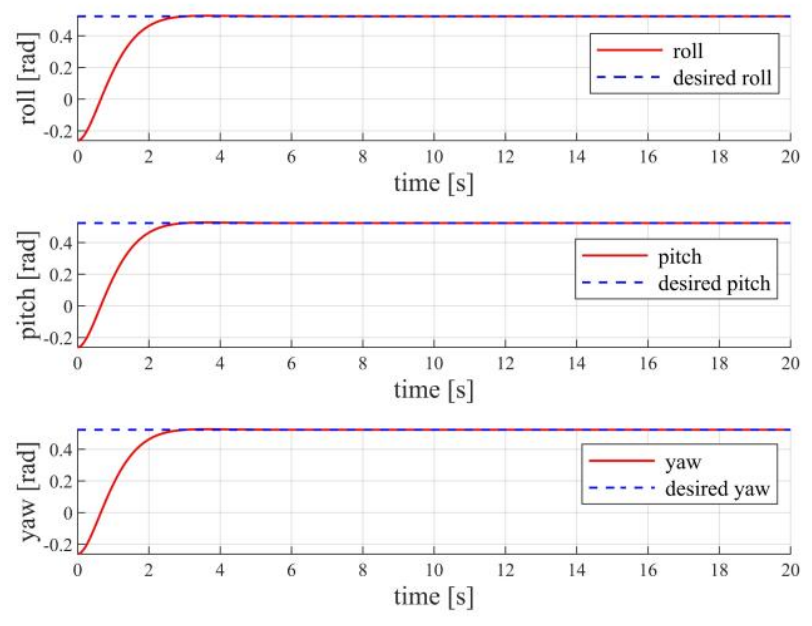

Fig. 5. Attitude control responses
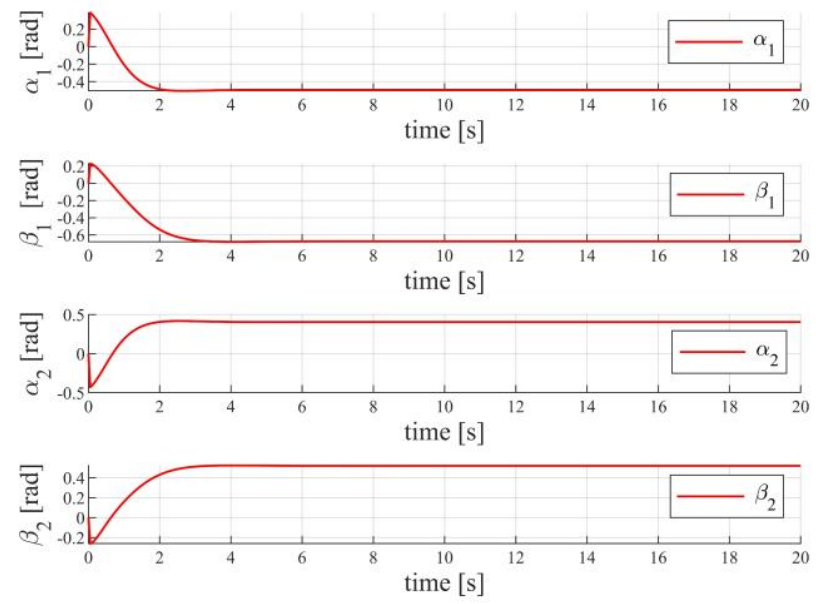

Fig. 6. Tilted angle responses
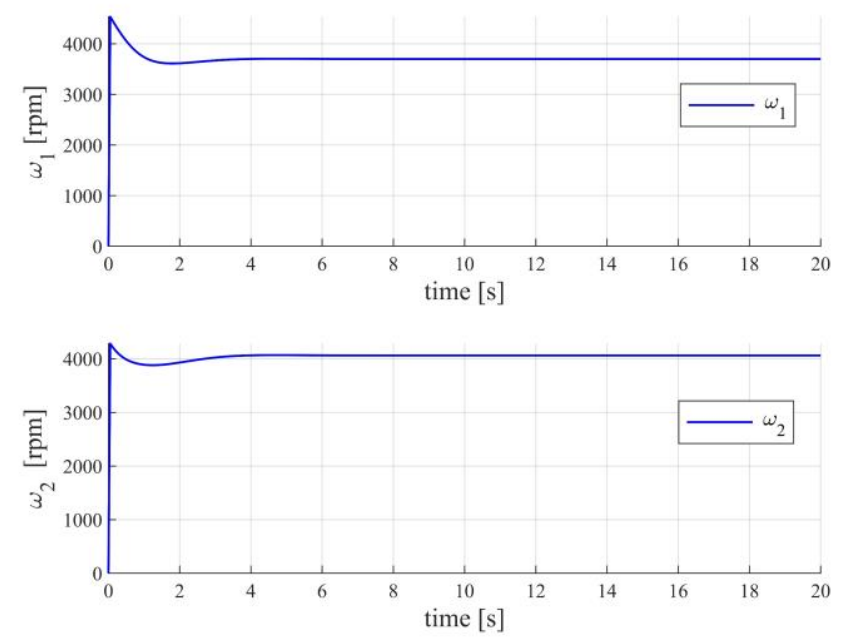

Fig. 7. Rotational speed responses

\section{B. Simulation 2: Tracjectory Tracking Problem}

In the trajectory tracking simulation, a 3D space trajectory is set to $\boldsymbol{X}_{d}(t)=\left[\begin{array}{llllll}x_{d}(t) & y_{d}(t) & z_{d}(t) & \frac{\pi}{6} & \frac{\pi}{6} & \frac{\pi}{6}\end{array}\right]^{T}$ and the initial state of the system is set to $\boldsymbol{X}_{0}=\left[\begin{array}{llllll}0 & 0 & 0 & 0 & 0 & 0\end{array}\right]^{T}$, where the trajectory is defined as below:

$$
\left\{\begin{array}{c}
x_{d}(t)=0.5 \sin \left(\frac{\pi}{8} t\right) \\
y_{d}(t)=0.5 \cos \left(\frac{\pi}{4} t\right) \\
z_{d}(t)=-1.0-0.5 \sin \left(\frac{\pi}{8} t\right)
\end{array}\right.
$$

where $t$ denotes the time.

Fig. 8 shows the position responses of the UAV robot in the trajectory simulation, where the position amplitudes of the $\mathrm{X}_{\mathrm{w}^{-}}, \mathrm{Y}_{\mathrm{w}^{-}}$and $\mathrm{Z}_{\mathrm{w}}$-axis are about $0.4988 \mathrm{~m}, 0.4766 \mathrm{~m}$ and $-0.5015 \mathrm{~m}$. The phase-shifts of the position responses in the $\mathrm{X}_{\mathrm{W}}-, \mathrm{Y}_{\mathrm{w}^{-}}$and $\mathrm{Z}_{\mathrm{w}}$-axis are about $0.3925 \mathrm{rad}, 0.7136 \mathrm{rad}$ and $0.3925 \mathrm{rad}$, respectively. And the delay of each axis is about $1.0 \mathrm{~s}, 1.1 \mathrm{~s}$ and $1.0 \mathrm{~s}$, respectively. The attitude responses of the UAV robot are converged to the desired values are shown 
in Fig. 9, where the rise time of the attitude responses around the $\mathrm{X}_{\mathrm{w}}{ }^{-}, \mathrm{Y}_{\mathrm{w}}-$ and $\mathrm{Z}_{\mathrm{w}}$-axis are all about $1.576 \mathrm{~s}$. And their overshoots around the $\mathrm{X}_{\mathrm{w}}{ }^{-}, \mathrm{Y}_{\mathrm{w}}{ }^{-}$and $\mathrm{Z}_{\mathrm{w}}$-axis are all about $0.0026 \mathrm{rad}$. The settling times of the attitude responses in the $\mathrm{X}_{\mathrm{w}^{-}}, \mathrm{Y}_{\mathrm{w}^{-}}$and $\mathrm{Z}_{\mathrm{w}}$-axis are all about $2.358 \mathrm{~s}$.

As shown in Fig. 10, the directions of the tilt angles $\alpha_{1}$ and $\beta_{1}$ are opposite to those of $\alpha_{2}$ and $\beta_{2}$. The time histories of all the tilted angles are changed in oscillatory modes after about $3.1 \mathrm{~s}$. Fig. 11 shows the rotational speeds of the tiltable coaxial rotors. It should be noted that the actuators used in reality will not respond to such speeds, because the rotor dynamics are ignored in this simulation.

In addition, Fig. 12 shows the trajectory in 3D space, where the errors between the desired trajectory and the current trajectory are found to be small.

From the simulation results, it is confirmed that the designed backstepping control strategy is both effective for the position and attitude control during the trajectory tracking simulation. It is also confirmed that the UAV robot is in a stable flight and the body of the UAV robot is also kept in the desired attitude state during the simulation.
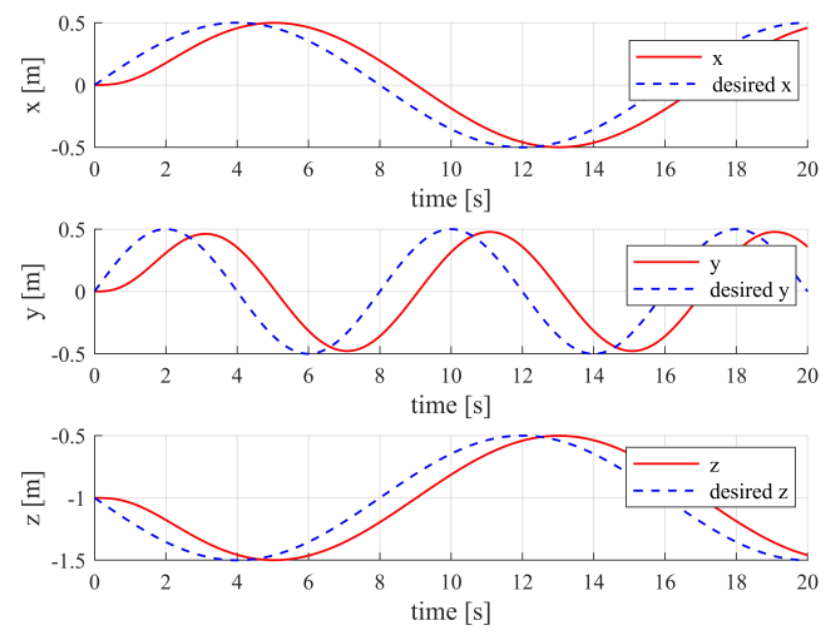

Fig. 8. Position control responses in trajectory tracking
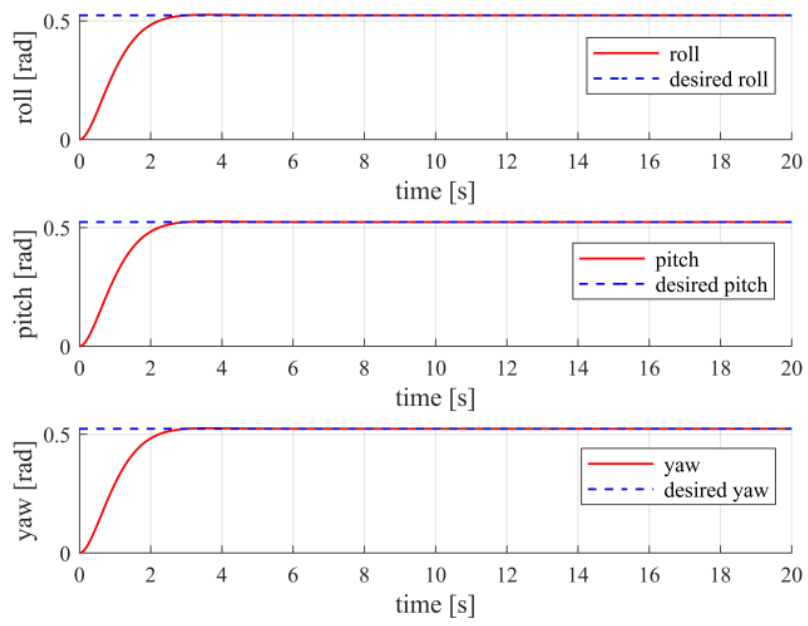

Fig. 9. Attitude control responses in trajectory tracking
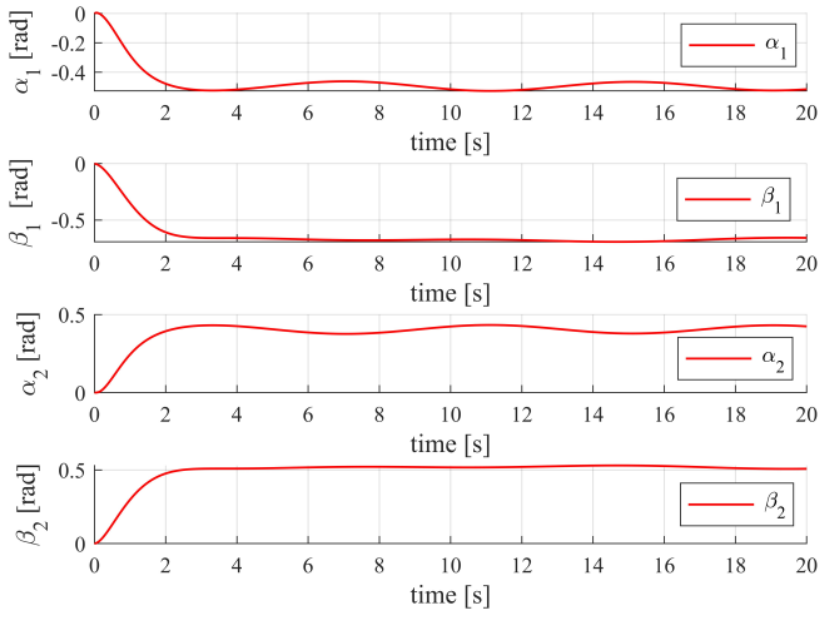

Fig. 10. Tilted angle responses in trajectory tracking
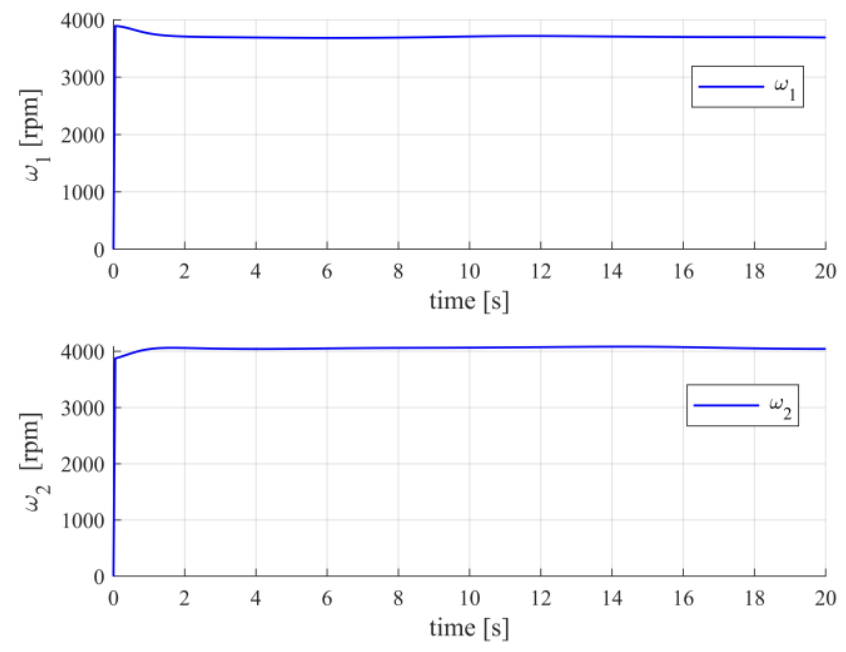

Fig. 11. Rotational speed responses in trajectory tracking

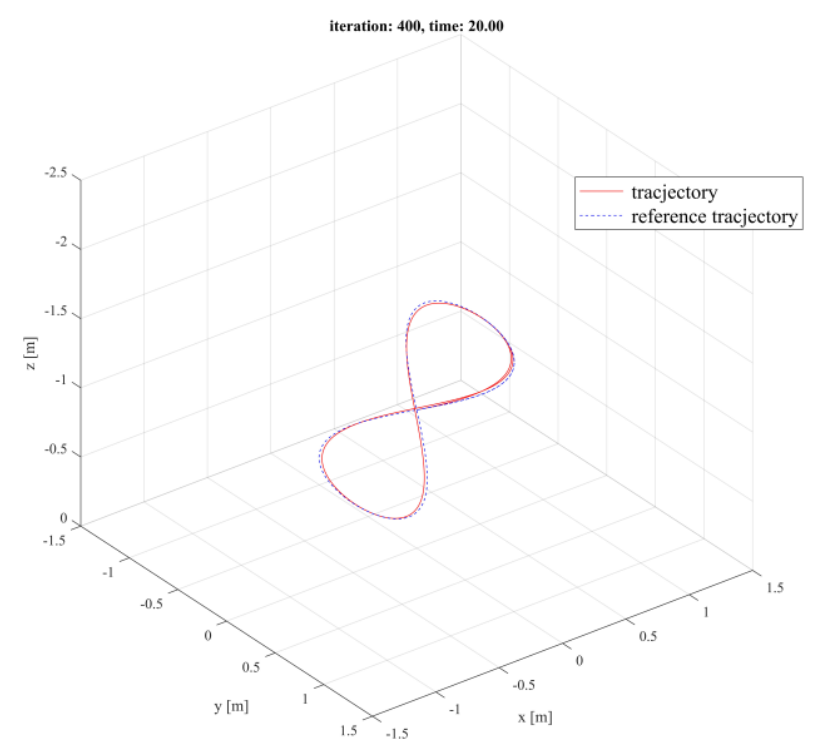


Fig. 12. The trajectory in 3D space

\section{CONCLUSIONS}

In this paper, a backstepping control method has been described for a tandem rotor UAV robot, in which each rotor has a tilt mechanism in two DOFs. Especially, an MIMO vectoral backstepping approach was adopted here, because the input distribution matrix was fortunately a square and nonsingular matrix. The related static control allocation problem was also solved by using a Moore-Penrose pseudoinverse. Two kinds of simulation of the UAV robot without wind disturbances were given to demonstrate the performance of the proposed control method.

As future works, it is going to be compared with other control strategies when considering other feedback linearization, wind disturbances, etc. Some real flight or motion tests also need to be conducted in the future.

\section{REFERENCES}

[1] H. Menouar, I. Guvenc, K. Akkaya, A. S. Uluagac, A. Kadri and A. Tuncer, "UAV-enabled intelligent transportation systems for the smart city: Applications and challenges," IEEE Commun. Mag., vol. 55, no. 3, pp. 22-28, 2017.

[2] M.R. Haque, M. Muhammad, D. Swarnaker, M. Arifuzzaman, "Autonomous quadcopter for product home delivery," in Proc. of International Conference on Electrical Engineering and Information \& Communication Technology (ICEEICT), 2014.

[3] K.T. San, E.Y. Lee, Y.S. Chang, "The delivery assignment solution for swarms of UAVs dealing with multi-dimensional chromosome representation of genetic algorithm," in Proc. of Ubiquitous Computing, Electronics \& Mobile Communication Conference (UEMCON), IEEE Annual, 2016.

[4] T. Tomic, K. Schmid, P. Lutz, A. Domel, M. Kassecher, E. Mair, I. L. Grixa, F. Ruess, M. Suppa, D. Burschka, "Toward a fully autonomous UAV: Research platform for indoor and outdoor urban search and rescue," IEEE Robotics and Automation Magazine, vol. 19, no. 3, pp. 46-56, 2012.

[5] J. Scherer, S. Yahyanejad, S. Hayat, E. Yanmaz, T. Andre, A. Khan, et al., "An autonomous multi-UAV system for search and rescue," in Proc. of Intern. Conf. Mobile Systems Applications and Services, pp. $1-6,2015$.

[6] A. Al-Kaff, F. M. Moreno, L. J. San José, F. García, D. Martín, A. De La Escalera, et al., "Vbii-uav: Vision-based infrastructure inspectionuav," in Proc. of World Conference on Information Systems and Technologies WorldCist'17, pp. 221-231, 2017.

[7] M. Nakao, M. Ito, Y. Kutuna, M. Yamada, M. Yamada and Y. Hada, "Development of a bridge inspection support robot system that uses a two-wheeled quad-rotor helicopter," in Proc. of East Asia-Pacific Conference on Structural Engineering and Construction, pp. 293-301, 2016.

[8] C. Zhang and J. M. Kovacs, "The application of small unmanned aerial systems for precision agriculture: A review," Precis. Agricult., vol. 13, no. 6, pp. 693-712, 2012.

[9] S. Khanal, J. Fulton and S. Shearer, "An overview of current and potential applications of thermal remote sensing in precision agriculture," Comput. Electron. Agricult., vol. 139, pp. 22-32, 2017.

[10] K. Whitehead, B. J. Moorman and C. H. Hugenholtz, "Low-cost ondemand aerial photogrammetry for glaciological measurement," Cryosphere Discuss., vol. 7, no. 3, pp. 1879-1884, 2013.

[11] Turner, D., Lucieer, A. and Wallace, L. (2013), "Direct georeferencing of ultrahigh-resolution UAV imagery," IEEE Transactions on Geoscience and Remote Sensing, available online doi: 10.1109/TGRS.2013.2265295. https://doi.org/10.1109/TGRS.2013.2265295

[12] C.H. Hugenholtz, K. Whitehead, O.W. Brown, T.E. Barchyn, B.J. Moorman, A. LeClair, K. Riddell, T. Hamilton, "Geomorphological mapping with a small unmanned aircraft system (sUAS): Feature detection and accuracy assessment of a photogrammetrically-derived digital terrain model," Geomorphology, vol. 194, pp. 16-24, 2013.

[13] H.C.T.E. Fernando, A. Silva, M. Zoysa and R. Munasinghe, "Modelling simulation and implementation of a quadrotor UAV," in Proc. of IEEE International Conference on Industrial and Information Systems, pp. 207-212, 2013.

[14] R. Rashad, J. Goerres, R. G. Aarts, J. B. Engelen, and S. Stramigioli, "Fully actuated multirotor UAVs: A literature review," IEEE Robot. Automat. Mag., doi: 10.1109/MRA.2019.2955964.

[15] M. Hamandi, F. Usai, Q. Sable, N. Staub, M. Tognon, and A. Franchi, "Survey on aerial multirotor design: a taxonomy based on input allocation," Jan. 2020, working paper or preprint. [Online]. Available: https://hal.archives-ouvertes.fr/hal-02433405

[16] K. Bodie, Z. Taylor, M. Kamel and R. Siegwart, "Towards efficient full pose omnidirectionality with overactuated MAVS," 2018, [online] Available: https://arxiv.org/abs/1810.06258.

[17] S. Rajappa, M. Ryll, H. H. Bülthoff and A. Franchi, "Modeling control and design optimization for a fully-actuated hexarotor aerial vehicle with tilted propellers," in Proc. of 2015 IEEE Int. Conf. Robotics and Automation (ICRA), pp. 4006-4013, 2015.

[18] Y. Tadokoro, T. Ibuki, and M. Sampei, "Classification and structural evaluation of fully-actuated hexrotor UAVs," in Proc. Annu. Amer. Control Conf. (ACC), pp. 1945-1950, 2018.

[19] G. Jiang, R. Voyles, K. Sebesta, and H. Greiner, "Estimation and optimization of fully-actuated multirotor platform with nonparallel actuation mechanism," in Proc. 2017 IEEE/RSJ Int. Conf. Intelligent Robots and Systems (IROS), pp. 6843-6848. doi: 10.1109/IROS.2017.8206605.

[20] A. Franchi, R. Carli, D. Bicego and M. Ryll, "Full-pose tracking control for aerial robotic systems with laterally bounded input force," IEEE Trans. Robot., vol. 34, no. 2, pp. 534-541, 2018.

[21] M. Allenspach, K. Bodie, M. Brunner, L. Rinsoz, Z. Taylor, M.Kamel, R. Siegwart and J. Nieto, "Design and optimal control of a tiltrotor micro aerial vehicle for efficient omnidirectional flight," arXiv preprint arXiv:2003.09512.

[22] P. Segui-Gasco, Y. Al-Rihani, H.S. Shin, and A. Savvaris, "A novel actuation concept for a multi rotor UAV," Journal of Intelligent \& Robotic Systems, vol. 74, no. 1-2, pp.173-191, 2014.

[23] X. Xu, K. Watanabe, and I. Nagai, "Development of an UAV robot that has multifunctional locomotion modes with tilted coaxial rotors," in Proc. of 2018 37th Chinese Control Conference (CCC). Wuhan, pp. 7896-7900, 2018.

[24] A. Bin Junaid, A. Diaz De Cerio Sanchez, J. Betancor Bosch, N. Vitzilaios, and Y. Zweiri, "Design and implementation of a dual-axis tilting quadcopter," Robotics, vol. 7, no. 4, pp. 65, 2018.

[25] M.J. Gerber, T. Tsao, "Twisting and tilting rotors for high-efficiency, thrust-vectored quadrotors," Journal of Mechanisms and Robotics, vol. 10, no. 6, pp. 061013, 2018.

[26] N. Amiri, A. Ramirez-Serrano, and R.J. Davies, "Integral backstepping control of an unconventional dual-fan unmanned aerial vehicle," J Intell Robot Syst, vol. 69, pp. 147-159, 2013.

[27] R. Mokhtari, C. Braham, B. Cherki, "Extended state observer based control for coaxial-rotor UAV," ISA Transactions, vol 61, pp. 1-14, 2014.

[28] J. Buzzatto, M. Liarokapis, "An agile, coaxial, omnidirectional rotor module: on the development of hybrid, all terrain robotic rotorcrafts," in Proc. of IEEE International Symposium on Safety, Security, and Rescue Robotics (SSRR), Abu Dhabi, 2020, unpublished.

[29] X. Xu, K. Watanabe and I. Nagai, "Feedback linearization control for a tandem rotor UAV robot equipped with two 2-DOF tiltable coaxialrotors," Artif Life Robotics (2020). https://doi.org/10.1007/s10015020-00655-X.

[30] M. Kamel, S. Verling, O. Elkhatib, C. Sprecher, P. Wulkop, Z. Taylor, R. Siegwart, and I. Gilitschenski, "The voliro omniorientational hexacopter: An agile and maneuverable tiltable-rotor aerial vehicle," IEEE Robotics \& Automation Magazine, vol. 25, no. 4, pp. 34-44, 2018.

[31] B. Li, D. Wang and L. Ma, "BioTetra: a bioinspired multi-rotor aerial vehicle," in Proc. of IEEE Int. Conf. on Robotics and Biomimetics (ROBIO), Dali, China, pp. 114-119, 2019. 
[32] L. Zhou, J. Zhang, H. She and H. Jin, "Quadrotor UAV flight control via a novel saturation integral backstepping controller," Automatika, vol. 60, no. 2, pp. 193-206, 2019.
[33] T. I. Fossen and J. P. Strand, "A tutorial on nonlinear backstepping: Applications to ship control," Model. Identification Contr., vol. 20, no. 2 , pp. 83-135, 1999, [online] Available: http://www.micjournal.no/micarchives.asp. 